\title{
Efecto de la Suplementación de Butirato de Sodio en la Dieta de Cuyes (Cavia porcellus) de Engorde sobre el Desarrollo de las Vellosidades Intestinales y Criptas de Lieberkühn
}

\author{
Effect of Sodium Butyrate Supplementation on Fattening Guinea Pig (Cavia \\ porcellus) Diets on the DeVelopment of INTESTINAL Villi and Crypts of Lieberkühn \\ Diego Vallejos P. ${ }^{1}$, Fernando Carcelén C. ${ }^{1,5}$, Ronald Jiménez A. ${ }^{4}$, Rosa Perales C. ${ }^{2}$, \\ Gilberto Santillán A. ${ }^{2}$, Miguel Ara G. ${ }^{1}$, William Quevedo ${ }^{3}$, Fernando Carzola ${ }^{1}$
}

\section{Resumen}

\begin{abstract}
El objetivo del presente estudio fue evaluar el efecto de la suplementación de butirato de sodio sobre el desarrollo de vellosidades intestinales y criptas de Lieberkühn en cuyes de engorde. Se utilizaron 45 cuyes machos destetados de 14 días de edad distribuidos en cinco tratamientos con nueve repeticiones por tratamiento: T1 fue el control, T2 recibió zinc-bacitracina (200 ppm) y T3, T4 y T5 fueron suplementados con 100, 200 y 300 ppm de butirato de sodio, respectivamente. Se evaluó el largo y ancho de las vellosidades intestinales, la profundidad de criptas de Lieberkühn y la relación entre el largo de vellosidad y profundidad de cripta (L/P) en duodeno, yeyuno e íleon a los 84 días de edad. Para el largo de vellosidades intestinales, T5 $(0.812 \mathrm{~mm})$ fue mayor a T1 $(0.701 \mathrm{~mm})$ en duodeno; asimismo, T5 y T4 fueron mayores a T3 ( $<<0.05)$. Para el ancho de vellosidades en el íleon T3 (0.114), T4 (0.115) y T5 $(0.136 \mathrm{~mm})$ fueron superiores a T1 y T2 (p<0.05). La profundidad de la cripta intestinal a nivel del yeyuno e íleon fue menor para T4 y T5 frente a T1 (p<0.05). Para L/P, T4 y T5 fueron mayores a T0 en los tres segmentos intestinales $(\mathrm{p}<0.05)$. La dieta suplementada con butirato de sodio afectó positivamente al desarrollo intestinal de cuyes de engorde con mejores resultados que una dieta base.
\end{abstract}

Palabras clave: cuy, butirato de sodio, desarrollo intestinal, morfometría intestinal

\footnotetext{
${ }^{1}$ Laboratorio de Bioquímica, Nutrición y Alimentación Animal, ${ }^{2}$ Laboratorio de Histología, Embriología y Patología Veterinaria, ${ }^{3}$ Fundo El Taro, Facultad de Medicina Veterinaria, Universidad Nacional Mayor de San Marcos, Lima, Perú

${ }^{4}$ Estación Experimental del Centro de Investigaciones IVITA - El Mantaro, Universidad Nacional Mayor de San Marcos, Junín, Perú

${ }^{5}$ E-mail: nandodeme@gmail.com
} 
The aim of this study was to evaluate the effect of sodium butyrate supplementation on the development of intestinal villi and crypts of Lieberkühn in guinea pigs during the fattening period. A total of 45 male guinea pigs of 14 days of age (weaning age) were distributed in five treatments with nine repetitions per treatment: T1 was the control, T2 received zinc-bacitracin (200 ppm), and T3, T4 and T5 were supplemented with 100, 200 and $300 \mathrm{ppm}$ of sodium butyrate respectively. The length and width of the villi, crypt of Lieberkühn depth and villus length and crypt depth ratio (L/D) in the duodenum, jejunum and ileum were evaluated at 84 days of age. In the length of intestinal villi, the duodenum T5 $(0.812 \mathrm{~mm})$ was larger than T1 $(0.701 \mathrm{~mm})$, also, T5 and T4 were larger than T3 ( $\mathrm{p}<0.05)$. The width of ileum villi in T3 $(0.114)$, T4 $(0.115)$ and T5 $(0.136 \mathrm{~mm})$ were wider than T1 and $\mathrm{T} 2$ ( $\mathrm{p}<0.05)$. The depth of the intestinal crypt in jejunum and ileum was smaller in T4 and $\mathrm{T} 5$ than $\mathrm{T} 1(\mathrm{p}<0.05)$. The L/D ratio showed that T4 and T5 was higher than T0 in the three intestinal segments $(\mathrm{p}<0.05)$. The diet supplemented with sodium butyrate positively affected the intestinal development of guinea pigs during the fattening period as compared to the basal diet.

Key words: guinea pig, sodium butyrate, intestinal development, intestinal morphometry

\section{INTRODUCCIÓN}

Los ácidos orgánicos, particularmente los de cadena corta, mejoran el rendimiento productivo cuando se usan como aditivos en la dieta de animales de engorde (Santomá et al., 2006). Actúan como agentes tróficos de los enterocitos al comportarse como una fuente rápida de energía, donde favorecen la regeneración del epitelio intestinal y promueven el desarrollo morfométrico intestinal (Camino y Fernández, 2005). El efecto integral ha sido muchas veces el aumento en el ritmo de crecimiento (Carro y Ranilla, 2002).

El ácido butírico, usado comúnmente como sal sódica, es un ácido orgánico de cadena corta con efectos a nivel molecular, celular y tisular (Gálfi, 2011). El butirato de sodio es considerado como un inhibidor de la deacetilasa de histonas (HDAC's) (Domokos et al., 2010; Garczarczyk et al., 2010), como estimulador de la síntesis de proteína, tanto de colágeno como de no-colágeno, en la mucosa (Lan et al., 2005) y como regulador de los niveles de las citoquinas IL-8 y IL-6 en el intestino durante procesos inflamatorios (Evans et al., 2005). El butirato es reconocido por su efecto directo sobre la secreción de mucina $y$, principalmente, por su efecto antibacteriano sobre enteropatógenos Gram negativos como Escherichia coli y Salmonella sp, y Gram positivos como Clostridium sp (Sánchez et al., 2011), por lo que se le considera como alternativa al uso de antibióticos promotores del crecimiento.

El modo de acción del butirato en la proliferación celular intestinal está probablemente asociado a un aumento de la disponibilidad de un sustrato energético. Se ha demostrado en ratas, borregos y humanos como fuentes energéticas en orden de importancia al butirato, acetoacetato, glutamina y glucosa (Gutiérrez, 1998; De las Cagigas y Blanco, 2002); asimismo, se ha demostrado, in vivo, el potencial del butirato para acidificar el tubo digestivo (Santomá et al., 2006). Además, tiene la propiedad de pasar de la forma disociada a la no disociada, dependiendo del $\mathrm{pH}$ del medio, convirtiéndolo en un eficaz agente antimicrobiano (Santomá et al., 2006). 
Estudios de suplementación en lechones y gallinas han demostrado que el butirato provoca un mayor crecimiento de las vellosidades intestinales y una menor profundidad de las criptas de Lieberkühn del epitelio intestinal (Kotunia et al., 2004 y Sánchez et al., 2009, respectivamente). Considerando estos resultados, el presente estudio tuvo como objetivo evaluar el efecto de la suplementación de la dieta de cuyes de engorde con butirato de sodio para determinar su efecto sobre las vellosidades intestinales y las criptas de Lieberkühn.

\section{Materiales y MÉTodos}

La fase de campo se realizó entre febrero y marzo de 2013 en la Estación Experimental El Mantaro del Centro de Investigaciones IVITA, ubicada en el distrito de El Mantaro, departamento de Junín, Perú, a 3320 msnm. La zona presenta una temperatura media anual de $10.9{ }^{\circ} \mathrm{C}$ y precipitación pluvial anual de $750 \mathrm{~mm}$. La evaluación histológica se realizó en el Laboratorio de Histología, Embriología y Patología Veterinaria de la Facultad de Medicina Veterinaria, Universidad Nacional Mayor de San Marcos, Lima.

Se trabajó con 45 cuyes machos obtenidos por cruzamiento entre la línea Prolífica y la línea Lechera (Jiménez y Huamán, 2010). Los cuyes tenían 14 días de edad, estaban recién destetados y fueron distribuidos al azar en cinco tratamientos que recibieron 0,100 , 200,300 ppm de butirato de sodio o $200 \mathrm{ppm}$ de zinc-bacitracina como suplemento a una dieta base.

Como fuente de zinc-bacitracina se usó Promozinb ${ }^{\circledR} 10 \%$ (Laboratorios CUSA, Perú). La dosis recomendada por el fabricante para pollos, pavos y cerdos es de 50 ppm. En este estudio se usó 200 ppm, dosis dos veces mayor a la usada, sin efecto alguno, por Bazay et al. (2014) en cuyes. Como fuente de butirato de sodio se utilizó Gustor
BP70® (butirato de sodio al 70\% parcialmente protegido) (NOREL Animal Nutrition, España). El butirato de sodio y el zincbacitracina correspondiente a cada tratamiento fue mezclado previamente con lotes de 40 $\mathrm{kg}$ de afrecho, de acuerdo a lo recomendado por Behnke (1992), donde primero se mezcló el butirato con $5 \mathrm{~kg}$ de afrecho y luego se incorporó la mezcla a los $35 \mathrm{~kg}$ restantes de afrecho en una mezcladora horizontal por 5 minutos.

La dieta base estuvo compuesta por concentrado de afrechillo de trigo y forraje obtenido por corte de un plantel de Rye grass cv. Tama (Lolium multiflorum) más trébol rojo (Trifolium pratense) con una proporción de siembra 3:1. El forraje ofrecido por día fue equivalente al $50 \%$ del peso vivo y contenía $20 \%$ de materia seca (MS), $18 \%$ de proteína cruda (PC) y 2.7 Mcal de energía digestible (ED) por kilogramo de MS. El afrecho de trigo contenía $90 \%$ de MS, $16 \%$ de PC y 2.65 Mcal ED/kg MS. La proporción forraje/concentrado fue aproximadamente de 80:20. El manejo de los animales se realizó de acuerdo a los procedimientos rutinarios para animales de engorde de la Granja de Cuyes del IVITA, excepto que los cuyes fueron alojados en pozas individuales de $0.36 \mathrm{~m}^{2}$, con completa independencia de alimentación y espacio vital.

Los cuyes se sacrificaron a los 84 días de edad mediante inhalación rápida de cloroformo y degüello. Se extrajo el intestino delgado y se obtuvieron muestras de $1 \mathrm{~cm}$ de largo del duodeno (a $3 \mathrm{~cm}$ del píloro), yeyuno (sección media de las asas intestinales) e éleon (a $3 \mathrm{~cm}$ de la unión ileocecal). Las muestras se fijaron en formol bufferado $10 \%$ por $24 \mathrm{~h}$. Las muestras fueron reducidas a porciones de 4 a $5 \mathrm{~mm}$ de largo. Luego fueron lavadas y deshidratadas con alcohol etílico al 70\%, aclaradas con xilol, e incluidas en parafina para obtener cortes transversales de la mucosa intestinal de $5 \mathrm{~mm}$ de espesor. Las láminas resultantes fueron teñidas con hematoxilina-eosina. 


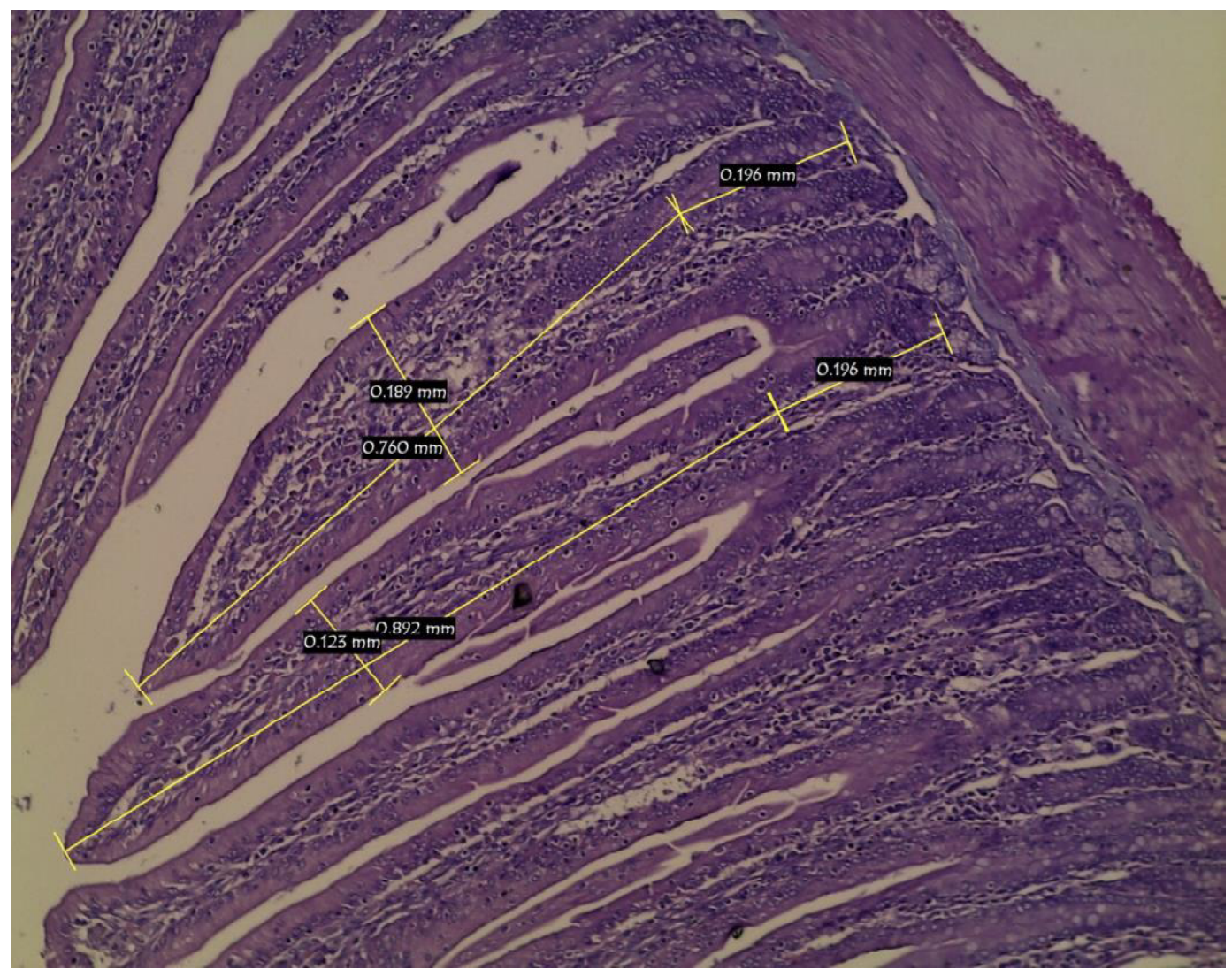

Figura 1. Corte histológico de duodeno de cuy a 100x de aumento. Se muestra los puntos de referencia para la medición del largo y ancho de vellosidad intestinal y la profundidad de cripta de Lieberkühn

Para las mediciones del largo y ancho de las vellosidades intestinales y la profundidad de las criptas (en mm) se siguió un protocolo adaptado de Olivera et al. (2000) y Zhang et al. (2005). En cada corte histológico se estableció de manera virtual entre 8 a 10 campos cuadrangulares que abarcaron toda la superficie del corte en una vista de 40x. En cada campo y a 100x de aumento se realizaron las mediciones morfométricas en las vellosidades íntegras (con epitelio continuo, completas, que aparezcan íntegramente dentro del campo) y en sus respectivas criptas de Lieberkühn. Se obtuvieron los promedios de cada segmento intestinal correspondiente a cada animal.
Las mediciones se facilitaron con el uso del programa LAS EZ Leica ${ }^{\circledR}$ instalado en una computadora conectada a un microscopio Leica ${ }^{\circledR}$ DM500, que incluía una cámara Leica ${ }^{\circledR}$ ICC50. El sistema permitió determinar la distancia entre cualquier par de puntos fijados por el usuario. El largo de la vellosidad fue medido desde el ápice de la misma hasta el ápice de la entrada de la cripta de Lieberkühn. El ancho de la vellosidad fue medida como una línea perpendicular a la sección media de la vellosidad. La profundidad de cripta de Lieberkühn fue medida desde la entrada a la cripta hasta la zona basal de la misma (Fig. 1). 
Cuadro 1. Efecto de la suplementación de butirato de sodio sobre el largo de vellosidad (mm) en el duodeno, yeyuno e íleon de cuyes de engorde a los 84 días de edad

\begin{tabular}{lccccc}
\hline \multirow{2}{*}{ Sección } & \multirow{2}{*}{ Control } & \multirow{2}{*}{ Antibiótico } & \multicolumn{3}{c}{ Butirato de Sodio } \\
\cline { 4 - 6 } & & & $100 \mathrm{ppm}$ & $200 \mathrm{ppm}$ & $300 \mathrm{ppm}$ \\
\hline Duodeno & $0.701^{\mathrm{bc}}$ & $0.733^{\mathrm{bac}}$ & $0.677^{\mathrm{c}}$ & $0.770^{\mathrm{ba}}$ & $0.812^{\mathrm{a}}$ \\
Yeyuno & $0.499^{\mathrm{a}}$ & $0.506^{\mathrm{a}}$ & $0.521^{\mathrm{a}}$ & $0.556^{\mathrm{a}}$ & $0.562^{\mathrm{a}}$ \\
Íleon & $0.457^{\mathrm{a}}$ & $0.469^{\mathrm{a}}$ & $0.438^{\mathrm{a}}$ & $0.493^{\mathrm{a}}$ & $0.502^{\mathrm{a}}$ \\
\hline
\end{tabular}

a,b,c Superíndices diferentes dentro de filas indica diferencia estadística $(p \varangle 0.05)$

Cuadro 2. Efecto de la suplementación de butirato de sodio sobre el ancho de vellosidad (mm) en el duodeno, yeyuno e íleon de cuyes de engorde a los 84 días de edad

\begin{tabular}{lccccc}
\hline \multirow{2}{*}{ Sección } & \multirow{2}{*}{ Control } & \multirow{2}{*}{ Antibiótico } & \multicolumn{3}{c}{ Butirato de Sodio } \\
\cline { 4 - 6 } & & & $100 \mathrm{ppm}$ & $200 \mathrm{ppm}$ & $300 \mathrm{ppm}$ \\
\hline Duodeno & $0.118^{\mathrm{a}}$ & $0.121^{\mathrm{a}}$ & $0.122^{\mathrm{a}}$ & $0.126^{\mathrm{a}}$ & $0.131^{\mathrm{a}}$ \\
Yeyuno & $0.105^{\mathrm{a}}$ & $0.107^{\mathrm{a}}$ & $0.109^{\mathrm{a}}$ & $0.110^{\mathrm{a}}$ & $0.111^{\mathrm{a}}$ \\
Íleon & $0.099^{\mathrm{c}}$ & $0.101^{\mathrm{c}}$ & $0.114^{\mathrm{b}}$ & $0.115^{\mathrm{b}}$ & $0.136^{\mathrm{a}}$ \\
\hline
\end{tabular}

a,b,c Superíndices diferentes dentro de filas indica diferencia estadística $(p \varangle 0.05)$

Se estimó el efecto global de los niveles de butirato de sodio y del antibiótico sobre el largo y ancho de vellosidades intestinales, la profundidad de cripta de Lieberkühn y la razón longitud/profundidad (L/P) mediante un análisis de varianza correspondiente a un diseño completamente al azar con cinco tratamientos y nueve repeticiones (cuyes). Las diferencias entre los niveles del butirato de sodio y el antibiótico fueron analizadas mediante la prueba de Duncan. El nivel de significancia para todas las pruebas de hipótesis fue de 0.05. Para el análisis estadístico se usó el paquete SAS/STAT ${ }^{\circledR}$ (SAS Institute Inc., 2009).

\section{Resultados}

A nivel del duodeno, el tratamiento con $300 \mathrm{ppm}$ de butirato de sodio produjo vellosidades más largas que el control y que el tratamiento con $100 \mathrm{ppm}$ de butirato de sodio $(\mathrm{p}<0.05)$, pero fueron estadísticamente similares al tratamiento con antibiótico y al suplementado con 200 ppm de butirato de sodio. Para las secciones del yeyuno e íleon, los tratamientos no mostraron diferencias significativas (Cuadro 1).

Los niveles crecientes de butirato de sodio y el uso de zinc-bacitracina no tuvieron 
Cuadro 3. Efecto de la suplementación de butirato de sodio sobre la profundidad de la cripta intestinal (mm) en el duodeno, yeyuno e íleon de cuyes de engorde a los 84 días de edad

\begin{tabular}{lccccc}
\hline \multirow{2}{*}{ Sección } & \multirow{2}{*}{ Control } & \multirow{2}{*}{ Antibiótico } & \multicolumn{3}{c}{ Butirato de Sodio } \\
\cline { 4 - 6 } & & & $100 \mathrm{ppm}$ & $200 \mathrm{ppm}$ & $300 \mathrm{ppm}$ \\
\hline Duodeno & $0.236^{\mathrm{a}}$ & $0.230^{\mathrm{a}}$ & $0.222^{\mathrm{a}}$ & $0.217^{\mathrm{a}}$ & $0.218^{\mathrm{a}}$ \\
Yeyuno & $0.230^{\mathrm{a}}$ & $0.205^{\mathrm{ab}}$ & $0.199^{\mathrm{ab}}$ & $0.191^{\mathrm{b}}$ & $0.188^{\mathrm{b}}$ \\
Íleon & $0.230^{\mathrm{a}}$ & $0.218^{\mathrm{ab}}$ & $0.200^{\mathrm{ab}}$ & $0.183^{\mathrm{b}}$ & $0.179^{\mathrm{b}}$ \\
\hline
\end{tabular}

$a, b, c$ Superíndices diferentes dentro de filas indica diferencia estadística $(p \varangle .05)$

Cuadro 4. Efecto de la suplementación de butirato de sodio sobre la relación entre el largo de vellosidad y la profundidad de cripta intestinal en el duodeno, yeyuno e îleon de cuyes de engorde a los 84 días de edad

\begin{tabular}{lccccc}
\hline \multirow{2}{*}{ Sección } & \multirow{2}{*}{ Control } & \multirow{2}{*}{ Antibiótico } & \multicolumn{3}{c}{ Butirato de Sodio } \\
\cline { 4 - 6 } & & & $100 \mathrm{ppm}$ & $200 \mathrm{ppm}$ & $300 \mathrm{ppm}$ \\
\hline Duodeno & $3.017^{\mathrm{c}}$ & $3.253^{\mathrm{bac}}$ & $3.081^{\mathrm{bc}}$ & $3.563^{\mathrm{ba}}$ & $3.7234^{\mathrm{a}}$ \\
Yeyuno & $2.263^{\mathrm{c}}$ & $2.489^{\mathrm{bc}}$ & $2.672^{\mathrm{abc}}$ & $2.941^{\mathrm{ba}}$ & $3.123^{\mathrm{a}}$ \\
Íleon & $2.060^{\mathrm{b}}$ & $2.186^{\mathrm{b}}$ & $2.255^{\mathrm{b}}$ & $2.745^{\mathrm{a}}$ & $2.864^{\mathrm{a}}$ \\
\hline
\end{tabular}

${ }_{a, b, c}$ Superíndices diferentes dentro de filas indica diferencia estadística $(p \varangle 0.05$ )

un efecto significativo en el ancho de vellosidades a nivel del duodeno y yeyuno. A nivel del íleon, los tratamientos con butirato de sodio mostraron una mejor respuesta que los grupos control o con zinc-bacitracina. La mejor respuesta se observó en el tratamiento con 300 ppm de butirato de sodio $(\mathrm{p}<0.05)$ (Cuadro 2).

Ninguno de los tratamientos tuvo un efecto significativo sobre la profundidad de cripta a nivel del duodeno. No obstante, a nivel del yeyuno e íleon se observaron mejores resultados para los tratamientos con $200 \mathrm{y}$
$300 \mathrm{ppm}$ de butirato de sodio frente al grupo control $(\mathrm{p}<0.05)$ (Cuadro 3).

La razón L/P a nivel del duodeno y yeyuno fue mayor para los tratamientos con 200 y 300 ppm de butirato frente al grupo control. Asimismo, el tratamiento con $300 \mathrm{ppm}$ de butirato obtuvo una mayor $\mathrm{L} / \mathrm{P}$ que el tratamiento con $100 \mathrm{ppm}(\mathrm{p}<0.05)$. A su vez, el tratamiento con $300 \mathrm{ppm}$ de butirato de sodio obtuvo una mayor L/P en yeyuno que zincbacitracina. En el íleon, la L/P fue mayor para los tratamientos con 200 y 300 ppm de butirato de sodio con respecto a 0 y $100 \mathrm{ppm}$ y al antibiótico (Cuadro 4). 


\section{Discusión}

A los 84 días de edad, la suplementación con 200 o 300 ppm de butirato de sodio produjo vellosidades más largas en el tramo del duodeno, vellosidades más anchas en el íleon y criptas de Lieberkühn más profundas en el yeyuno e íleon, lo cual aumenta la razón L/P, denotando un tejido más maduro.

El uso de ácidos orgánicos de cadena corta, como el butírico, en la alimentación de lechones, aves, conejos y ratas causa un aumento del desarrollo de las vellosidades intestinales, gracias a su efecto sobre el ambiente luminal, a su acción bacteriostáticobactericida y a su acción directa sobre los enterocitos (Camino y Fernández, 2005). Galfi y Bokori (1990), al adicionar $0.17 \%$ de sales de ácido butírico en la dieta de lechones obtuvieron un aumento sustancial en el número de las células constituyentes de las vellosidades intestinales, así como en su longitud a nivel del íleon. Se ha demostrado, además, que los ácidos orgánicos producidos por la fermentación microbiana de carbohidratos en el lechón (ácido acético, propiónico y n-butírico) estimulan la proliferación celular intestinal (Gutiérrez, 1998; Partanen y Mroz, 1999).

Un incremento de la razón L/P, producto de vellosidades más largas y criptas menos profundas denota una mayor capacidad de digestión y absorción a nivel de intestino delgado (Rubio et al., 2010). Los tratamientos suplementados con butirato de sodio produjeron un tejido intestinal con mayor $\mathrm{L} / \mathrm{P}$ frente al tratamiento con zinc-bacitracina, lo que sugiere que, en el contexto de madurez y funcionalidad intestinal, los ácidos orgánicos se comportan mejor o igual que los antibióticos promotores del crecimiento. Este supuesto es apoyado por los resultados de Sánchez-Silva et al. (2014) quienes concluyen que los ácidos orgánicos pueden reemplazar eficientemente a los promotores de crecimiento, tipo antibiótico, en la alimentación de cuyes de crecimiento y por Sánchez et al. (2011), quie- nes encontraron comportamientos similares entre butirato de sodio con 300 ppm y zincbacitracina en gallinas semipesadas IsaBabcock B-380 de 32 semanas de edad.

La bacitracina tiene efectos antibióticos sobre la microflora intestinal, explicados por la disminución de la competencia por los nutrientes entre bacterias favorables y patógenas, así como por la disminución de metabolitos microbianos que afectan el crecimiento (Ortiz, 2004). La bacitracina en conejos y pollos de engorde produce una reducción del grosor de las paredes y un adelgazamiento de las vellosidades intestinales, en parte por una menor proliferación de las células de la mucosa debido a la disminución de ácidos grasos de cadena corta derivados de fermentación microbiológica (Furlan et al., 2002; Ortiz, 2004). Esto explicaría la ligera disminución de los valores morfométricos intestinales en los animales suplementados con este antibiótico en el presente estudio.

Aunque no son consistentes, los niveles de butirato de sodio en estudio muestran diferencias estadísticas respecto al nivel de 0 $\mathrm{ppm}$. Esta tendencia fue similar a los resultados de Rico y Sans (1993) en conejos de engorde. Por su lado, Sánchez et al. (2009), en un estudio con gallinas Bovans blanca, compararon niveles de 0,300 y $500 \mathrm{ppm}$ de butirato de sodio, encontrando las mejores respuestas en producción y calidad del cascarón con los niveles de 300 y 500 ppm. Estas referencias y los resultados del presente experimento permiten sustentar una relación directa entre la cantidad de butirato de sodio y el desarrollo intestinal.

\section{Conclusiones}

- La suplementación con butirato de sodio en la dieta de cuyes de engorde genera mayor desarrollo en el largo y ancho de vellosidades intestinales, así como en la profundidad de la cripta, especialmente en la dosis de 300 ppm. 
- La suplementación con butirato de sodio fue superior o similar al uso de zincbacitracina como antibiótico promotor de crecimiento.

\section{Literatura Citada}

1. Bazay G, Carcelén F, Ara M, Jiménez R, González R, Quevedo W. 2014. Efecto de los manano-oligosacáridos sobre los parámetros productivos de cuyes (Cavia porcellus) durante la fase de engorde. Rev Inv Vet Perú 25: 198204. doi: $10.15381 /$ rivep.v25i2.8491

2. Behnke DC. 1992. Como mezclar alimentos de calidad: perspectivas sobre uniformidad de mezclado. Soya Noticias 229: 6-12.

3. Camino T, Fernández S. 2005. Ácidos orgánicos en primeras edades. Albéitar 88: 64-66.

4. Carro MD, Ranilla MJ. 2002. Los aditivos antibióticos promotores del crecimiento de animales: situación actual y posibles alternativas. [Internet]. Disponible en: http://www.produccionanimal.com.ar/informacion_tecnica/ invernada_promotores_crecimiento/01aditivos_antibioticos_promotores.pdf

5. De las Cagigas L, Blanco E. 2002. Prebióticos y probióticos, una relación beneficiosa. Rev Cub Aliment Nutr 16: 63-68.

6. Domokos M, Jakus J, Szeker K, Csizinszky R, Csiko GY, Neogrady ZS, Csordas A, Galfi P. 2010. Butyrateinduced cell death and differentiation are associated with distinct patterns of ROS in HT29-derived human colon cancer cells. Dig Dis Sci 55: 920-930. doi: 10.1007/s10620-009-0820-6

7. Evans M, Tian J, Gu L, Jones D, Ziegler TR. 2005. Dietary supplementation with orotate and uracil increases adaptive growth of jejunal mucosa after massive small bowel resection in rats/ discussant/author's response. J Parenter Enteral Nutr 29: 315-320. doi: 10.1177/ 0148607105029005315
8. Furlan AC, Scapinello C, Moreira I, Martins EN, Murakami AE, Buranelo FL. 2002. Cobre e bacitracina de zinco como promotores de crescimento em rações para coelhos em crescimento. Maringá 24: 1027-1030. doi: 10.4025/ actascianimsci.v24i0.2520

9. Gálfi P. 2011. Prevención de enfermedades infecciosas en avicultura por medio de aditivos. Bol Téc $\mathrm{N}^{\circ} 3$ NOREL Animal Nutrition. $12 \mathrm{p}$.

10. Gálfi P, Bokori J. 1990. Feeding trial in pigs with a diet containing sodium nbutyrate. Acta Vet Hung 38(1-2): 3-17.

11. Garczarczyk D, Szeker K, Galfi P, Csordas A, Hofmann J. 2010. Protein kinase $C \tilde{a}$ in colon cancer cells: expression, $\mathrm{Thr}^{514}$ phosphorylation and sensitivity to butyrate-mediated upregulation as related to the degree of differentiation. Chem Biol Interact 185: 25-32. doi: 10.1016/j.cbi.2010.02.035

12. Gutiérrez C. 1998. Los ácidos grasos de cadena corta en un centro de investigaciones en Norteamérica. AMMVEPE 9(1): 9-12.

13. Jiménez R, Huamán R. 2010. Manual para el manejo de reproductores híbridos especializados en producción de carne. El Mantaro, Perú: INCAGROACRICUCEN-UNMSM. 175 p.

14. Kotunia A, Wolinski J, Laubitz D, Jurkowska M, Romé D, Guilloteau P, Zabielski R. 2004. Effect of sodium butyrate on the small intestine development in neonatal piglets fed by artificial sow. J Physiol Pharmacol 55(Suppl 2): 59-68.

15. Lan Y, Verstegen MWA, Tamminga S, Williams BA. 2005. The role of the commensal gut microbial community in broiler chickens. World Poultry Sci J 61(1): 95-104. doi: 10.1079/WPS200445

16. Oliveira P, Murakami A, Garcia E, Macari M, Scapinello C. 2000. Influência de fatores antinutricionais da leucena (Leucaena leucocephala e Leucaena cunningan) e do feijão guandu (Cajanus cajan) sobre o epitélio intestinal e o desempenho de frangos de 
corte. Rev Bras Zootec 29: 1759-1769. doi: 10.1590/S1516-35982000000600024

17. Ortiz MP. 2004. Utilización de alternativas naturales a los antibióticos promotores del crecimiento en la salud intestinal y parámetros productivos de pollos broilers. Tesis de Ingeniero Agrónomo. Valparaíso, Chile: Univ Católica de Valparaíso. $107 \mathrm{p}$.

18. Partanen KH, Mroz Z. 1999. Organic acids for performance enhancement in pig diets. Nutr Res Rev 12: 117-145. doi: 10.1079/095442299108728884

19. Rico R, Sans E. 1993. Efecto de una formulación oral con ácidos grasos volátiles, en gazapos de engorde. Bol Cunicultura 66: 56-58.

20. Rubio LA, Ruiz R, Peinado MJ y Echavarri A. 2010. Morphology and enzymatic of the small intestinal mucosa of Iberian pigs as compared with a lean pig strain. J Anim Sci 88: 3590-3597. doi: 10.2527/jas.2010-3040

21. Sánchez I, Posadas E, Sánchez E, Fuente B, Laparra JL, Ávila E. 2011. Efecto de butirato de sodio sobre algunos parámetros productivos de gallinas de postura en semilibertad. Vet Méx 42: 227-232.
22. Sánchez I, Posadas E, Sánchez E, Fuente B, Laparra JL, Ávila E. 2009. El efecto del butirato de sodio en dietas para gallinas sobre el comportamiento productivo, calidad del huevo y vellosidades intestinales. Vet Méx 40: 397-403.

23. Sánchez-Silva M, Carcelén F, Ara M, Gonzáles R, Quevedo W, Jiménez R. 2014. Efecto de la suplementación de ácidos orgánicos sobre los parámetros productivos del cuy Rev Inv Vet Perú 25: 381-389. doi: 10.15381/rivep. v25i3.10116

24. Santomá G, Pérez de Ayala P, Guitiérrez del Álamo A. 2006. Producción de broilers sin antibióticos promotores de crecimientos actuales. En: LIII Symposium Científico de Avicultura. Barcelona, España.

25. SAS Institute Inc. 2009. SAS/STAT® 9.2 User's Guide. $2^{\text {nd }}$ ed. Cary, NC: SAS Institute Inc.

26. Zhang AW, Lee BD, Lee SK, Lee KW, Lee CH, An GH, Sough B. 2005. Effects of yeast (Saccharomyces cerevisiae) cell components on growth performance, meat quality, and ileal mucosa development of broiler chicks. Poultry Sci 84: 1015-1021. doi: 10.1093/ ps/84.7.1015 